\title{
Prognostic significance of L-type amino acid transporter 1 (LAT1) and 4F2 heavy chain (CD98) expression in surgically resectable stage III non-small cell lung cancer
}

\author{
KYOICHI KAIRA ${ }^{1}$, NOBORU ORIUCHI ${ }^{2}$, HISAO IMAI ${ }^{1}$, KIMIHIRO SHIMIZU $^{3}$, NORIKO YANAGITANI ${ }^{1}$, \\ NORIAKI SUNAGA ${ }^{1}$, TAKESHI HISADA ${ }^{1}$, OSAMU KAWASHIMA ${ }^{5}$, YOSUKE KAMIDE ${ }^{6}$, \\ TAMOTSU ISHIZUKA $^{1}$, YOSHIKATSU KANAI ${ }^{7}$, TAKASHI NAKAJIMA ${ }^{4}$ and MASATOMO MORI ${ }^{1}$ \\ Departments of ${ }^{1}$ Medicine and Molecular Science, ${ }^{2}$ Diagnostic Radiology and Nuclear Medicine, \\ ${ }^{3}$ Thoracic and Visceral Organ Surgery, and ${ }^{4}$ Tumor Pathology, Gunma University Graduate School of Medicine; \\ Departments of ${ }^{5}$ Surgery, and ${ }^{6}$ Respiratory Medicine, National Nishi-Gunma Hospital, Gunma; \\ ${ }^{7}$ Division of Bio-System Pharmacology, Department of Pharmacology, \\ Osaka University Graduate School of Medicine, Osaka, Japan
}

Received May 6, 2010; Accepted June 28, 2010

DOI: $10.3892 / \mathrm{etm} .2010 .117$

\begin{abstract}
The purpose of this study was to evaluate the prognostic value of L-type amino acid transporter 1 (LAT1) and 4F2 heavy chain (CD98) expression in patients with stage III non-small cell lung cancer (NSCLC). A total of 188 consecutive patients with pathologic stage III NSCLC were retrospectively reviewed. The expression of LAT1, CD98, Ki-67 labeling index, vascular endothelial growth factor (VEGF) as well as microvessel density (MVD) were evaluated immunohistochemically and correlated with the prognosis of patients after complete resection of the tumor. Positive expression of LAT1 and CD98 was noted in 58\% (109/188) and $50 \%(94 / 188)$ of the cases, respectively $(p=0.1473)$. A positive rate of LAT1 expression was significantly higher in squamous cell carcinoma (SQC) $(90 \%, 48 / 53)$ and large-cell carcinoma (LCC) $(100 \%, 12 / 12)$ than in adenocarcinoma (AC) $(40 \%, 49 / 123)$. Moreover, a positive rate of LAT1 with CD98 expression was also significantly higher in SQC (74\%, $39 / 53)$ and LCC $(75 \%, 9 / 12)$ than AC $(34 \%, 42 / 123)$. LAT1 expression was significantly higher in patients with mediastinal lymph node metastases than in patients without, and was significantly correlated with CD98, Ki-67 labeling index, VEGF and MVD. The 5-year survival rates of LAT1-positive and -negative patients and CD98-positive and -negative patients were 27.9 and $40.6 \%(\mathrm{p}=0.0033)$, respectively, and 24.1 and $43.6 \%(\mathrm{p}=0.0004)$, respectively. Multivariate analysis
\end{abstract}

Correspondence to: Dr Kyoichi Kaira, Department of Medicine and Molecular Science, Gunma University Graduate School of Medicine, Showa-machi, Maebashi, Gunma 371-8511, Japan E-mail: kkaira1970@yahoo.co.jp

Key words: L-type amino acid transporter 1, non-small cell lung cancer, stage III, 4F2 heavy chain confirmed that positive expression of LAT1 and CD98 was an independent factor predicting a poor prognosis. In conclusion, the overexpression of LAT1 and CD98 is a pathological factor for predicting the prognosis of patients with surgically resectable stage III NSCLC.

\section{Introduction}

Non-small cell lung cancer (NSCLC) is the leading cause of cancer-related death worldwide and is associated with poor patient prognosis (1). One of the most important prognostic factors is stage of disease. Since the last version of the International Staging System (ISS) was updated in 1997 (2,3), revision of this classification has been proposed by different authors (4-6). Particularly for stage III NSCLC, the 1997 ISS divides patients into stage IIIA and IIIB; this group of patients is heterogeneous, including those with surgically resectable tumors as well as those with unresectable tumors treated by chemotherapy and/or radiotherapy. Currently, increased attention is being focused on new biological parameters for predicting prognosis. Many biological markers have demonstrated independent prognostic significance in NSCLC, though not in every single study, and none have yet proven to be of clinical value (7).

Amino acid transporters are essential for growth and proliferation in normal and transformed cells $(8,9)$. The amino acid transport system $\mathrm{L}$ is a $\mathrm{Na}^{+}$-independent large neutral amino acid transport system $(8,10)$. L-type amino acid transporter 1 (LAT1) is one of the L-type amino acid transporters and transports large neutral amino acids, including leucine, isoleucine, valine, phenylalanine, tyrosine, tryptophan, methinine and histidine (10-12). LAT1 requires covalent association with the heavy chain of the 4F2 cell surface antigen (CD98) for its functional expression in the plasma membrane (10). Previous studies have shown LAT1 to be highly expressed in proliferating tissues, numerous tumor cell lines (T24 bladder 
carcinoma cells, RERF-LC-MA lung small-cell carcinoma cells and HeLa uterine cervical carcinoma cells) and primary human tumors (12). LAT1 expression was found to be closely related to the tumor cell growth of liver metastases in a rat model (13). Recent studies have demonstrated that positive expression of LAT1 is a significant factor for predicting poor prognosis in NSCLC and correlates with the grade of neuroendocrine tumors of the lung $(14,15)$. Nakanishi et al found that cooperative expression of LAT1 and CD98 was significantly correlated with both overall and disease-free survival rates in transitional cell carcinoma of the upper urinary tract (16). Nawashiro et al described that the overall immunoreactivity of LAT1 correlates well with the prognosis of patients with astrocytic tumors, and that high CD98 immunoreactivity also correlates with high LAT1 expression (17). However, it is unclear whether cooperative expression of LAT1 and CD98 is associated with overall survival in NSCLC. In our previous study, LAT1 expression was analyzed in a limited number of patients with stage III NSCLC (14). Thus, a large-scale study is required in order to assess the prognostic significance of LAT1 and CD98 expression in patients with stage III NSCLC.

As stage III NSCLC is a heterogeneous group, parameters other than disease stage must be examined in order to improve the therapeutic strategy and prognostic assessment. The purpose of the present study was to determine whether LAT1 and CD98 serve as significant prognostic factors, particularly in stage III NSCLC. In addition, LAT1 expression was correlated with the proliferative activity of the tumors as assessed by the $\mathrm{Ki}-67$ labeling index (LI) and with tumor angiogenesis as assessed by vascular endothelial growth factor (VEGF) expression, microvessel density (MVD) and the vascular invasiveness of the tumors.

\section{Materials and methods}

Patients. We analyzed 207 consecutive patients with stage III NSCLC who underwent resection either by lobectomy or pneumonectomy with mediastinal lymph node dissection at Gunma University Hospital and National Nishi-Gunma Hospital between June 1996 and December 2003. The involvement of mediastinal lymph nodes and malignant effusions was not detected pre-operatively in any of the patients. Nine patients who received induction chemotherapy or radiation therapy and 1 patient who died from a surgery-related complication were excluded. In 10 patients, no specimen was available for immunohistochemical analysis. Thus, a total of 188 patients (121 men, 67 women) were evaluated, including 52 cases of stage III disease involved in our previous study (14). The study protocol was approved by the institutional review board.

At the time of surgery, the age of the patients ranged from 36 to 80 years, with a mean age of 64 years. Histological classification according to the criteria of the World Health Organization revealed that 123 patients had adenocarcinoma (AD), 53 had squamous cell carcinoma (SQC) and 12 had large-cell carcinoma (LCC). Postoperative pathologic staging based on the current tumor-node-metastasis classification (3) revealed the tumors to be of stage IIIA $(n=114)$ and IIIB $(n=74)$. Postoperative adjuvant therapies in the form of platinumbased chemotherapy and radiation were administered to 6 and 8 patients, respectively. Intraoperative therapy was not performed in any of the patients. The postoperative clinical course was assessed by analyzing outpatient medical records and by telephone inquiries. The date of surgery was considered the start date for postoperative survival. The follow-up duration ranged from 6 to 125 months (mean 36).

\section{Immunohistochemical staining}

LAT1 and CD98. LAT1 expression was determined by immunohistochemical staining with an affinity-purified rabbit polyclonal anti-human LAT1 antibody (12). An oligopeptide corresponding to amino acid residues 497-507 of human LAT1 (CQKLMQVVPQET) was synthesized. The $\mathrm{N}$-terminal cysteine residue was introduced for conjugation with keyhole limpet hemocyanine. The antipeptide antibody was produced as described elsewhere (18). For immunohistochemical analysis, antiserum was affinity-purified as described previously (18).

Immunohistochemical staining was performed on paraffin sections using a polymer peroxidase method (Envision+/HRP; Dako Cytomation, Denmark). Briefly, deparaffinized rehydrated sections were treated with $0.3 \%$ hydrogen peroxide in methanol for 30 min to block endogenous peroxidase activity. To expose antigens, sections were autoclaved in $10 \mathrm{mmol} / \mathrm{l}$ sodium citrate buffer ( $\mathrm{pH}$ 6.0) for $5 \mathrm{~min}$ and cooled for $30 \mathrm{~min}$. After rinsing in $0.05 \mathrm{M}$ Tris-buffered saline containing $0.1 \%$ Tween-20, the sections were incubated with affinity purified anti-LAT1 antibody $(1.2 \mathrm{mg} / \mathrm{ml} ; 1: 3,200)$ overnight at $4^{\circ} \mathrm{C}$. The LAT1 antibody at a concentration of $0.375 \mu \mathrm{g} / \mathrm{ml}$ was used to stain for LAT1. Thereafter, they were incubated with Envision(+) rabbit peroxidase (Dako, Carpinteria, CA, USA) for $30 \mathrm{~min}$. The peroxidase reaction was performed using $0.02 \% 3,3$ '-diaminobenzidine tetrahydrochloride and $0.01 \%$ hydrogen peroxide in $0.05 \mathrm{~mol} / \mathrm{l}$ Tris- $\mathrm{HCl}$ buffer, $\mathrm{pH}$ 7.4. Finally, nuclear counterstaining was performed with Mayer's hematoxylin. For the negative control, the incubation step with the primary antibody was omitted. The specificity of immunoreactions using the anti-LAT1 antibody was established in previous studies $(17,19)$.

CD98 is an affinity purified goat polyclonal antibody raised against a peptide mapping at the carboxy terminus of CD98 of human origin. Immunohistochemical staining for CD98 was performed by the avidin-biotin method. Briefly, formalin-fixed and paraffin-embedded sections of resected specimens were dewaxed and rehydrated. The sections were incubated with affinity purified goat polyclonal antibody against CD98 (1:200; Santa Cruz Biotechnology, Inc., Santa Cruz, CA, USA) overnight at $4^{\circ} \mathrm{C}$.

LAT1 and CD98 expression were considered positive only when distinct membrane staining was present. Staining intensity was scored as follows: $1, \leq 10 \%$ of tumor area stained; $2,11-25 \%$ stained; $3,26-50 \%$ stained; $4, \geq 51 \%$ stained. The tumors in which stained tumor cells made up $>10 \%$ of the tumor were graded as positive. According to this scoring protocol, two investigators from among the authors, without prior knowledge of the clinical data, independently graded the staining intensity in all cases. To test the intraobserver variability, each section was reassessed by the same investigator after the first assessment was completed. The time interval between the first and second assessment was at least 
4 weeks. The interobserver variability was also determined by comparing the values of the first measurements of the two investigators.

$\mathrm{Ki}-67$. The detailed protocol for Ki-67 immunostaining was as published elsewhere (20). Briefly, formalin-fixed and paraffin-embedded sections of resected specimens were dewaxed, rehydrated, trypsinized and boiled in $0.01 \mathrm{~mol} / \mathrm{l}$ citrate buffer for $20 \mathrm{~min}$. For immunostaining, the murine monoclonal antibody MIB-1 (Dako, Denmark), specific for human nuclear antigen Ki-67, was used in a 1:40 dilution. The sections were lightly counterstained with hematoxylin. Sections of normal tonsil were used as a positive control for proliferating cells.

A highly cellular area of the immunostained sections was evaluated. All epithelial cells with nuclear staining of any intensity were defined as positive. Approximately 1,000 nuclei were counted on each slide. Proliferative activity was assessed as the percentage of MIB-1-stained nuclei (Ki-67 LI) in the sample.

$V E G F, C D 31$ and CD34. Immunohistochemical staining for VEGF, CD31 and CD34 was performed by the avidinbiotin method. In brief, sections were deparaffinized with xylene and rehydrated with ethanol. For VEGF, the sections were trypsinized and incubated with blocking serum. For CD31, antigen retrieval was carried out by placing the specimen in $0.01 \mathrm{~mol} / 1$ of citrate buffer at $\mathrm{pH} 6.0$ and then exposing it to microwave heating at $450 \mathrm{~W}$ for $20 \mathrm{~min}$. For CD34, the sections were treated by protease.

The antibodies used were: a monoclonal antibody against VEGF (1:100; Immuno-Biological Laboratories Co., Ltd., Japan); a mouse monoclonal antibody against CD31 (1:50; Dako); and a mouse monoclonal antibody against CD34 (1:200; Nichirei, Tokyo, Japan).

The expression of VEGF was quantitatively assessed according to the percentage of immunoreactive cells from a total of 1,000 neoplastic cells.

MVD was assessed using the criteria of Weidner et al (21). The areas of highest neovascularization were identified as regions of invasive carcinoma with the highest numbers of discrete microvessels stained for CD31 and CD34. Any brown-stained endothelial cell or endothelial cell cluster that was clearly separate from the adjacent microvessels, tumor cells and other connective tissue elements was considered a single countable microvessel. Microvessels in sclerotic areas within the tumor where microvessels were sparse as well as immediate adjacent areas of unaffected lung tissue were not considered in vessel counts. The number of CD31- and CD34positive vessels was counted in four selected hot spots in a x400 field $\left(0.26-\mathrm{mm}^{2}\right.$ field area). MVD was defined as the mean count of microvessels per $0.26-\mathrm{mm}^{2}$ field area (21).

Statistical analysis. The Mann-Whitney U test, paired two group t-test and $\chi^{2}$ test were used to examine the association of two categorical variables. Statistical analysis of LAT1 and CD98 scores was performed by the Mann-Whitney U test. In Ki-67 LI, VEGF and microvessel counts for CD31 and CD34, the paired two group t-test was performed. The Spearman rank-order correlation coefficient was used to assess the relationship between LAT1, Ki-67 LI, VEGF, MVD and other continuous variables.
The duration of survival was determined as the time from tumor resection to death from any cause. For survivors, the duration was determined according to the last date on which patients were known to be alive. The Kaplan-Meier method was used to estimate survival as a function of time, and differences in survival were analyzed by the log-rank test. Multivariate analyses were performed using the stepwise Cox proportional hazards model to identify independent prognostic factors. A p-value $<0.05$ was considered statistically significant. Statistical analysis was performed using StatView J-4.5 for Windows.

\section{Results}

Immunohistochemical findings. LAT1, CD98, Ki-67, VEGF, CD31 and CD34 immunohistochemical staining was evaluated for the 188 surgically resected primary lesions.

LAT1 and CD98. Expression of LAT1 and CD98 was localized predominantly on the plasma membrane of carcinoma cells in the tumor tissues (Fig. 1). Cytoplasmic staining was rarely evident. In the present study, no expression of LAT1 and CD98 was observed in any normal epithelial cells of the lung, including bronchial epithelium and alveolar cells. Positive LAT1 and CD98 expression was observed in 58\% (109/188) and $50 \%(94 / 188)$ of the samples, respectively $(\mathrm{p}=0.1473)$. The average score of LAT1 and CD98 expression was 2.1 1.1 and $1.8 \pm 0.9$, respectively $(\mathrm{p}=0.0113)$. A positive rate of LAT1 expression was significantly higher in SQC $(90 \%, 48 / 53)$ and LCC $(100 \%, 12 / 12)$ than in $\mathrm{AC}(40 \%, 49 / 123)$. A positive rate of LAT1 with CD98 expression was also significantly higher in SQC $(74 \%, 39 / 53)$ and LCC $(75 \%, 9 / 12)$ than in AC $(34 \%$, 42/123).

The expression of LAT1 according to clinicopathological parameters is shown in Table I. In NSCLC, LAT1 expression was significantly associated with gender, disease stage, lymph node metastases with N2-3 and vascular invasion. In AC, positive LAT1 expression was significantly associated with gender. In SQC, LAT1 expression was significantly associated with lymph node metastases with N2-3. In Table II, cooperative expression of LAT1 and CD98 (LAT1 with CD98) according to the clinicopathological parameters is listed. The cooperative expression of LAT1 with CD98 was also significantly associated with gender, disease stage and vascular invasion. N3 lymph node metastasis was observed in $2(1.6 \%)$ of 125 patients with N2-3. N2 lymph node metastasis was observed in 109 (96\%) of 114 patients with stage IIIA and $16(22 \%)$ of 74 patients with stage IIIB, demonstrating a significant difference $(\mathrm{p}<0.001)$.

$\mathrm{Ki}$-67. The median value of Ki-67 LI was $41 \%$ (range 2-85), and the value of $40 \%$ was established as the cutoff point. High expression ( $\mathrm{LI}>40 \%$ ) was noted in $95(51 \%)$ of 188 patients. The mean value of Ki-67 LI differed significantly between SQC $(53 \pm 14)$ and $\mathrm{AC}(31 \pm 18)(\mathrm{p}<0.001)$.

$V E G F, C D 31$, and $C D 34$. The staining pattern of VEGF was uniformly localized in the cytoplasm and/or membrane of neoplastic cells as shown in Fig. 1. The median rate of VEGF positivity was 35\% (range 1-90), and the value of $35 \%$ was established as the cutoff point. High expression $(>35)$ was noted in 97 (52\%) of 188 patients. The median rate of MVD as assessed by CD31 was 26 (range 1-69), and the value of $25 \%$ 
A

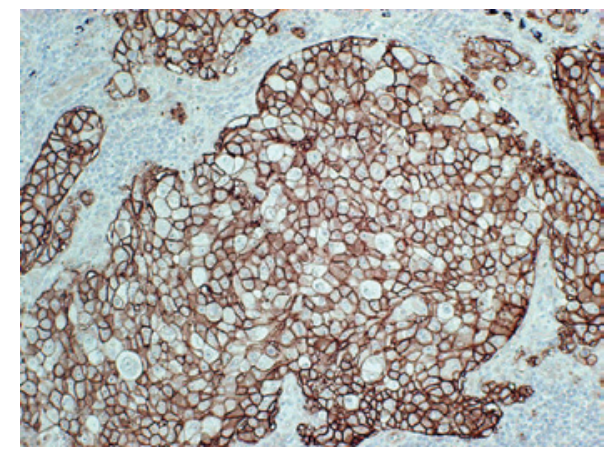

B

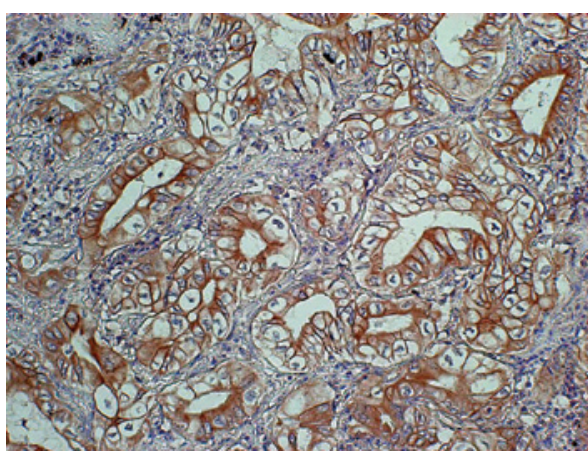

C

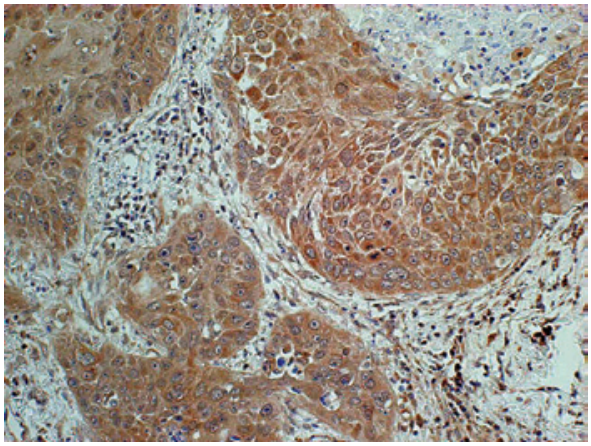

D

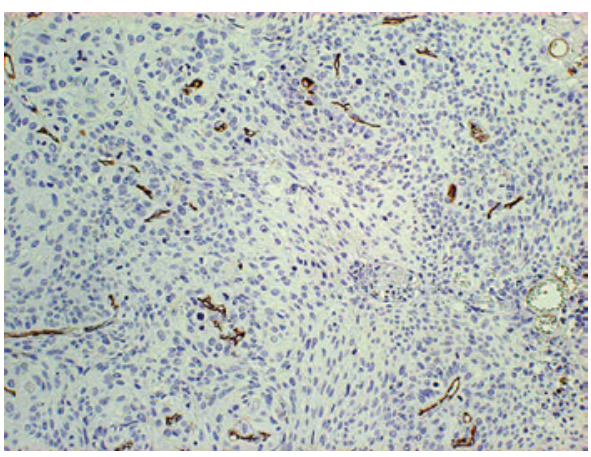

$\mathbf{E}$

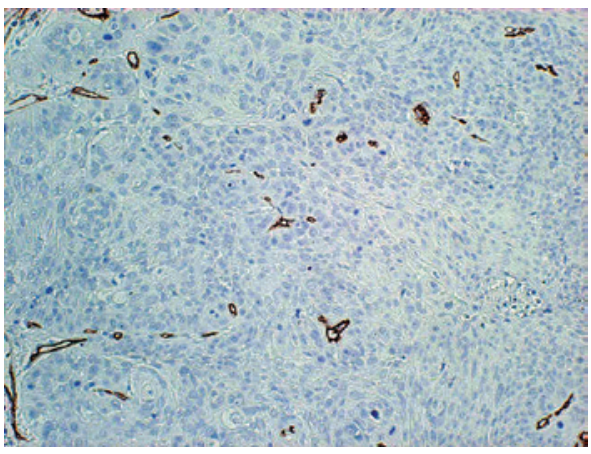

Figure 1. Immunohistochemical analysis in NSCLC. The scores for LAT1 (A) and CD98 (B) immunostaining were grade 4 and grade 4 , respectively, and the immunostaining pattern was membranous. Immunostaining for VEGF (C) showed that $>75 \%$ of the tumor cells were positive for anti-VEGF antibody. Immunostaining of CD31 (D) and CD34 (E) showed that many small vessels were positive for CD31 and CD34 in the stroma of the tumor tissue. was chosen as the cutoff point. High expression (>25) was noted in 103 (55\%) of 188 patients. The median rate of MVD as assessed by CD34 was 36 (range 2-121), and the value of $35 \%$ was chosen as the cutoff point. High expression $(>35)$ was noted in $99(53 \%)$ of 188 patients. The positive expression of VEGF, CD31 and CD34 did not differ significantly between SCC and AC. Analysis of the relationship between VEGF and the number of microvessels in the areas of the highest vascularization showed a significant association between the mean microvessel count and VEGF expression in the pulmonary lesions. VEGF expression was significantly associated with the number of microvessel counts [CD31-MVD $(\gamma=0.6218, p<0.0001)$ and CD34-MVD $(\gamma=0.6264, p<0.0001)$, respectively].

Correlation between LAT1, CD98, cell proliferation and angiogenesis. LAT1 expression was significantly correlated with Ki-67 LI, VEGF, CD31 and CD34 expression (Table III), and was closely correlated with CD98 expression $(\gamma=0.7320$, $\mathrm{p}<0.0001)$ and $\mathrm{Ki}-67 \mathrm{LI}(\gamma=0.7342, \mathrm{p}<0.0001)$. An association was also noted between LAT1 expression and histological type. In patients with AC, LAT1 expression was significantly correlated with Ki-67 LI, VEGF, CD31 and CD34, while in patients with SQC, it was significantly correlated with Ki-67 LI and CD98, but not with VEGF, CD31 and CD34.

Postoperative survival according to immunohistochemical markers. Postoperative survival was compared to the expression of LAT1 as shown in Table IV. For all patients, the 5 -year survival rates of the LAT1-positive and -negative patients were 27.9 and $40.6 \%$, respectively ( $\mathrm{p}=0.0033$, Fig. 2). Postoperative survival was also analyzed according to age, gender and pathologic stage. A significant difference in the prognosis between the LAT1-positive and -negative patients was demonstrated for the parameters: age $>65$ years, female and AC. Next, we analyzed the postoperative survival of LAT1 with CD98-positive patients and the other patients. For all patients, the 5-year survival rates of patients with cooperative LAT1 and CD98 positivity and of the other patients were 24.1 and $43.6 \%$, respectively ( $\mathrm{p}=0.0004$, Fig. 2 ). A significant difference in prognosis between LAT1 with CD98-positive patients and the other patients was demonstrated in terms of age, gender, pathologic stage and histology (Table V).

Postoperative survival was also analyzed according to the expression of Ki-67, VEGF, CD31 and CD34. For all patients, the 5-year survival rates of the Ki-67 LI-positive and -negative patients were 25.7 and $43.4 \%$, respectively $(\mathrm{p}=0.0006)$. No statistically significant difference in postoperative survival was observed in patients according to VEGF, CD31 and CD34 expression. Univariate analysis confirmed that positive expression of LAT1, LAT1 with CD98 and Ki-67 was a significant factor predicting poor prognosis. There was no significant difference in the 5-year survival rates according to age, gender, disease stage, histology, $\mathrm{N}$ factor, VEGF, CD31 and CD34.

Multivariate analysis of prognostic factors. Multivariate analysis confirmed that positive expression of LAT1 and CD98 was an independent factor for predicting poor prognosis (Table VI). Next, a multivariate analysis was performed 
Table I. Association between LAT1 expression and clinicopathological features.

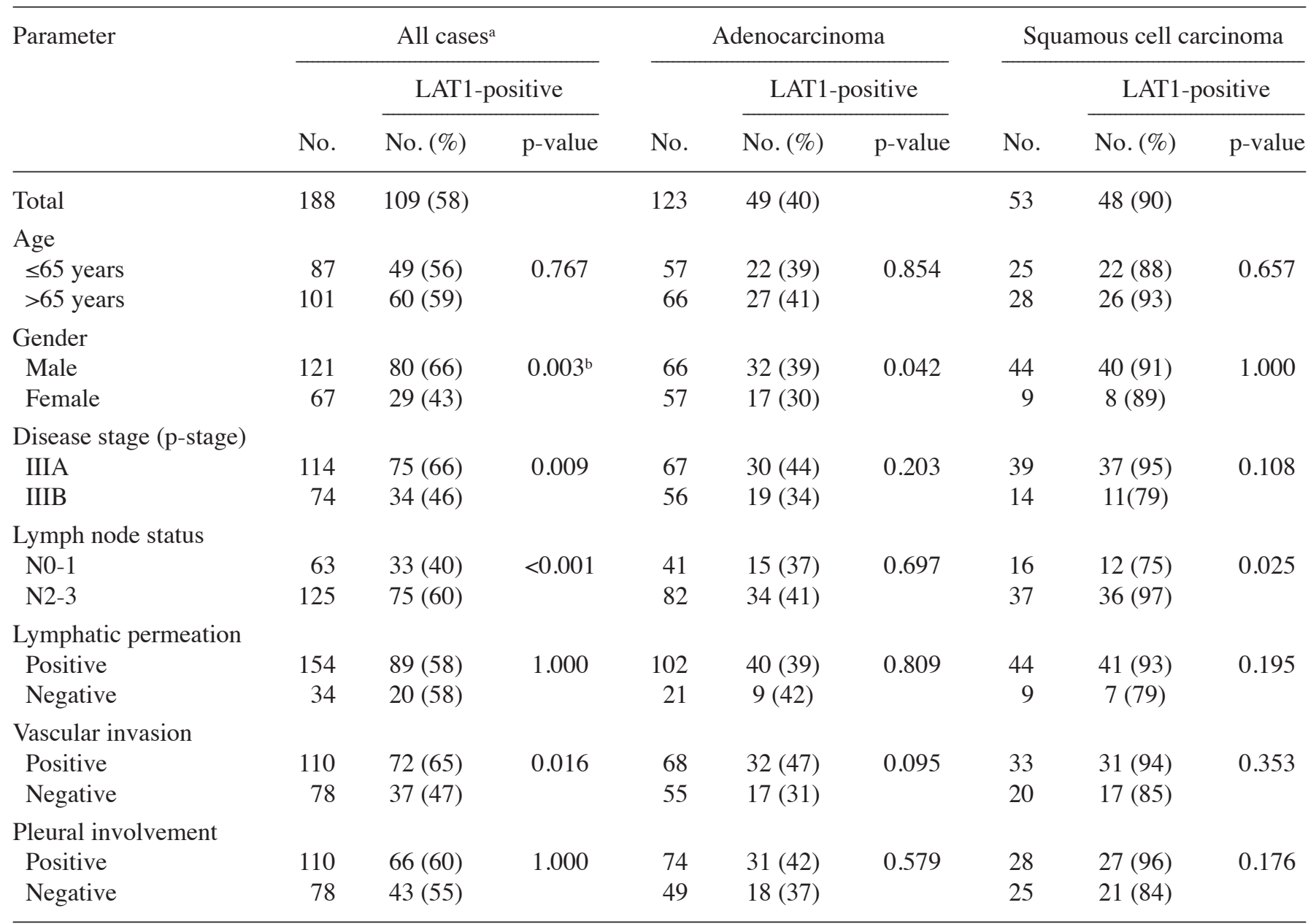

aLarge-cell carcinoma was included; 'bstatistically significant. LAT1, L-type amino acid transporter 1.

according to histological type. In $\mathrm{AC}$ patients, multivariate analysis confirmed that positive expression of LAT1 and CD98 was an independent factor for predicting poor prognosis. In SQC patients, multivariate analysis confirmed that positive expression of CD98 was an independent significant factor for predicting poor prognosis; however, the positive expression of LAT1 was not an independent prognostic factor.

\section{Discussion}

The present study evaluated the clinical significance of LAT1 and CD98 expression in surgically resected stage III NSCLC. The results of the study clearly demonstrate that the expression of LAT1 and CD98 is a significant independent factor for predicting poor prognosis in patients with surgically resected stage III NSCLC. LAT1 expression was higher in SQC than in AC. In patients with AC, the expression of LAT1 and CD98 was a significant independent prognostic factor. However, in patients with SQC, the expression of CD98 was a significant independent prognostic factor.

We recently reported that LAT1 expression was a significant prognostic factor in stage I-III NSCLC (14). In a previous study, the positive rate of LAT1 expression was $23 \%(38 / 164)$ in stage I AC and 88\% (61/69) in stage I SQC. Accordingly, the expression of LAT1 was higher in stage III as compared to stage I AC. However, the expression of LAT1 was equivalent between patients with stage I and III SQC. It is uncertain why the incidence of LAT1 expression was low in AC as compared to SQC. LAT1 expression was closely associated with cell proliferation and CD98 expression. The present study demonstrated that Ki-67 LI and CD98 were significantly higher in SQC than in AC. These factors may be associated with difference in the LAT1 expression profile of AC and SQC. On the other hand, one in vitro study demonstrated a link between amino acid transporter expression and mammalian target-ofrapamycin (mTOR) function (23). Although the mechanism of LAT1 or CD98 expression may be associated with the mTOR signaling pathway, this association was not examined in the present study. Further investigation of this association is warranted.

A previous study found LAT1 expression to be closely related to the tumor cell growth of liver metastases in a rat model (13). Tumor size in the LAT1 with CD98-positive group was significantly larger than tumor size in the other groups. Moreover, we previously reported that the expression of LAT1 and CD98 was significantly higher in the metastatic sites than the primary tumor sites of various human neoplasms (24). These results suggest that the overexpression of LAT1 
Table II. Association between cooperative expression of LAT1 with CD98 and clinicopathological features.

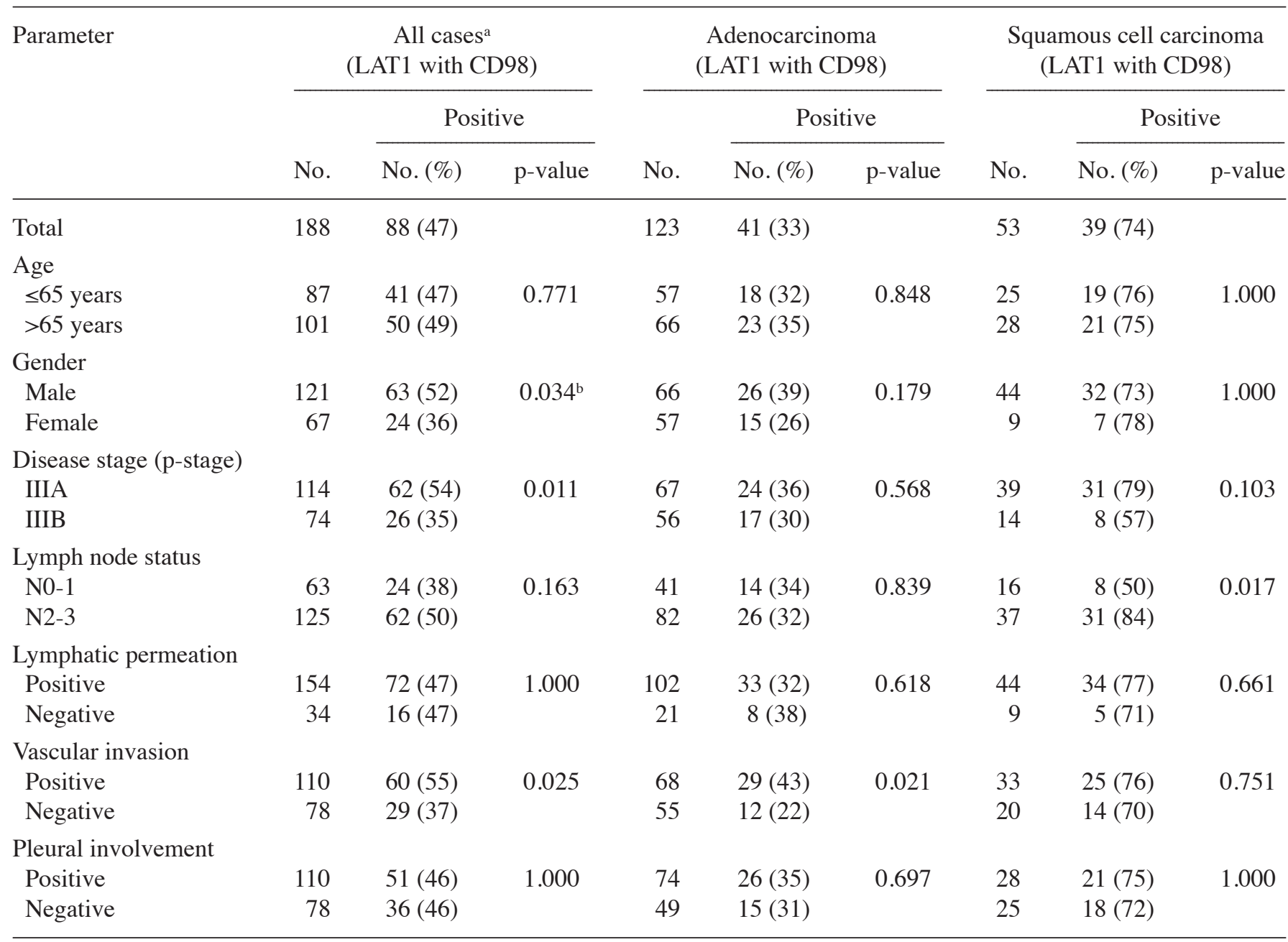

${ }^{\mathrm{a}}$ Large cell carcinoma was included; ${ }^{\mathrm{b}}$ statistically significant. LAT1, L-type amino acid transporter 1.

and CD98 plays an important role in the metastatic process of human neoplasms, including NSCLC. In the present study, the expression of LAT1 was significantly higher in patients with mediastinal lymph node metastases than in patients without mediastinal lymph node metastases. Since the incidence of mediastinal lymph node metastases was significantly higher in stage IIIA than in stage IIIB cases, LAT1 expression may be higher in stage IIIA than that in stage IIIB NSCLC (Tables I and II). Another previous study also demonstrated that LAT1 expression is significantly higher in NSCLC patients with lymph node metastases than in NSCLC patients without (14). The overexpression of LAT1 may play an important role in the metastatic process of lymph nodes, and may be correlated with patient prognosis.

CD98 is a disulphide-linked $125-\mathrm{kDa}$ heterodimeric membrane glycoprotein that is found on the cell surface of most normal cells. However, CD98 is also involved in cellular proliferation, transformation, fusion and adhesion, as well as in the LAT system, and its expression is up-regulated in a variety of tumors $(16,17,25,26)$. Ohkame et al reported the overexpression of CD98 in metastatic liver tumors in a rat model (13). In the present study, no expression of CD98 protein was observed in any normal epithelial cells of the lung, including the bronchial epithelium and alveolar cells. Overexpression of LAT1 with CD98 was more closely associated with poor prognosis than LAT1 expression alone. Notably, in SQC the coexpression of LAT1 and CD98 was an independent prognostic factor, while the expression of LAT1 was not noted. These results suggest that CD98 may play a significant role in tumor progression. Therefore, the up-regulation of both LAT1 and CD98 may be required for poor prognosis rather than that of LAT1 alone.

Amino acid transport systems play an important role in the regulation of cellular proliferation, whereas the details concerning its function as a promoter of tumor cell proliferation have not been clarified (11). Previous studies in addition to the present study indicate that LAT1 expression is significantly associated with $\mathrm{Ki}-67 \mathrm{LI}$, suggesting the role of LAT1 in cellular proliferation (14,27). Ki-67 LI was significantly higher in SQC and LCC than in AC. A meta-analysis revealed that the expression of $\mathrm{Ki}-67$ is a factor of poor survival prognosis in NSCLC (28). The present study also revealed that high Ki-67 LI was associated with an unfavorable prognosis in NSCLC patients. On the other hand, LAT1 expression was significantly associated with angiogenesis as determined by the expression of VEGF, CD31 and CD34 in AC, although 
Table III. Correlation between the expression of LAT1 and other immunohistochemical markers.

\begin{tabular}{|c|c|c|c|}
\hline Markers & Spearman $\gamma$ & $95 \%$ confidence interval & p-value \\
\hline \multicolumn{4}{|l|}{ CD98 } \\
\hline All cases ${ }^{a}(n=188)$ & 0.7320 & $0.6553-0.7937$ & $<0.0001$ \\
\hline $\mathrm{AC}(\mathrm{n}=123)$ & 0.7382 & $0.6424-0.8113$ & $<0.0001$ \\
\hline SQC $(n=53)$ & 0.5347 & $0.3016-0.7075$ & $<0.0001$ \\
\hline \multicolumn{4}{|l|}{ Ki-67 } \\
\hline $\operatorname{All}^{\mathrm{a}}(\mathrm{n}=188)$ & 0.7342 & $0.6580-0.7955$ & $<0.0001$ \\
\hline $\mathrm{AC}(\mathrm{n}=123)$ & 0.6616 & $0.5451-0.7530$ & $<0.0001$ \\
\hline $\mathrm{SQC}(\mathrm{n}=53)$ & 0.3632 & $0.0948-0.5824$ & 0.0075 \\
\hline \multicolumn{4}{|l|}{ VEGF } \\
\hline $\mathrm{All}^{\mathrm{a}}(\mathrm{n}=188)$ & 0.1442 & $0.0032-0.2854$ & 0.0484 \\
\hline $\mathrm{AC}(\mathrm{n}=123)$ & 0.2611 & $0.0828-0.4232$ & 0.0035 \\
\hline SQC $(n=53)$ & 0.2103 & $-0.0718-0.4612$ & 0.1307 \\
\hline \multicolumn{4}{|l|}{ CD31 } \\
\hline $\mathrm{All}^{\mathrm{a}}(\mathrm{n}=188)$ & 0.3073 & $0.1675-0.4349$ & $<0.0001$ \\
\hline $\mathrm{AC}(\mathrm{n}=123)$ & 0.3953 & $0.2285-0.5379$ & $<0.0001$ \\
\hline SQC $(n=53)$ & 0.1769 & $-0.1062-0.4335$ & 0.2050 \\
\hline \multicolumn{4}{|l|}{ CD34 } \\
\hline $\operatorname{All}^{\mathrm{a}}(\mathrm{n}=188)$ & 0.3590 & $0.2235-0.4808$ & $<0.0001$ \\
\hline $\mathrm{AC}(\mathrm{n}=123)$ & 0.4237 & $0.2617-0.5625$ & $<0.0001$ \\
\hline $\mathrm{SQC}(\mathrm{n}=53)$ & 0.2288 & $-0.0525-0.4764$ & 0.0994 \\
\hline
\end{tabular}

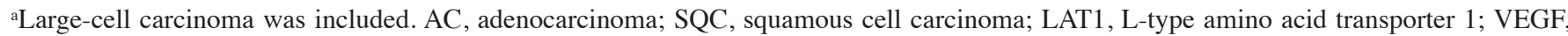
vascular endothelial growth factor.

Table IV. Five-year survival according to LAT1 expression.

\begin{tabular}{|c|c|c|c|}
\hline \multirow[t]{2}{*}{ Variable } & \multicolumn{3}{|c|}{ Five-year survival rate $(\%)^{\mathrm{a}}$} \\
\hline & LAT1-positive & LAT1-negative & $\mathrm{p}$-value ${ }^{\mathrm{b}}$ \\
\hline All patients & 27.9 & 40.6 & 0.0033 \\
\hline \multicolumn{4}{|l|}{ Age } \\
\hline$\leq 65$ years & 33.9 & 38.5 & 0.2583 \\
\hline$>65$ years & 27.0 & 42.5 & 0.0033 \\
\hline \multicolumn{4}{|l|}{ Gender } \\
\hline Male & 31.7 & 35.4 & 0.1987 \\
\hline Female & 23.6 & 46.6 & 0.0061 \\
\hline \multicolumn{4}{|l|}{ Pathologic stage } \\
\hline IIIA & 29.5 & 38.7 & 0.0412 \\
\hline IIIB & 25.1 & 43.1 & 0.0317 \\
\hline \multicolumn{4}{|l|}{ Histology } \\
\hline Adenocarcinoma & 23.3 & 38.7 & 0.0032 \\
\hline Squamous cell carcinoma & 33.0 & 60.0 & 0.1449 \\
\hline
\end{tabular}

aKaplan-Meier analysis; ${ }^{\mathrm{b}} \log$-rank test. LAT1, L-type amino acid transporter 1.

these markers were not factors of poor prognosis in NSCLC. In contrast to a previous study, whicch showed that the expression of VEGF is correlated with poor prognosis in stage I NSCLC (29), the present study revealed that neither
VEGF nor MVD were independent prognostic markers in stage III NSCLC.

Several clinical investigations have shown an increased uptake of radio-labelled amino acids in human neoplasms 
A

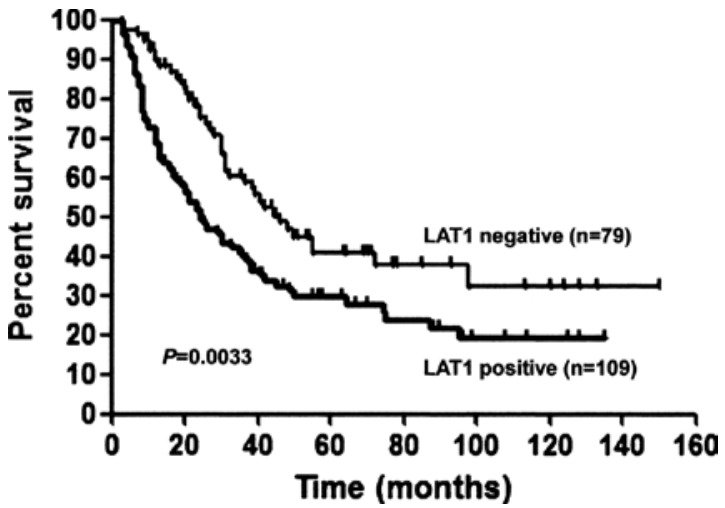

C

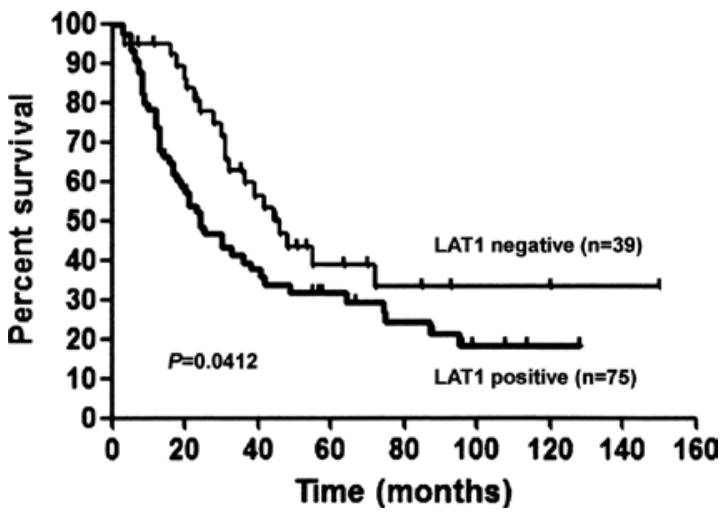

$\mathbf{E}$

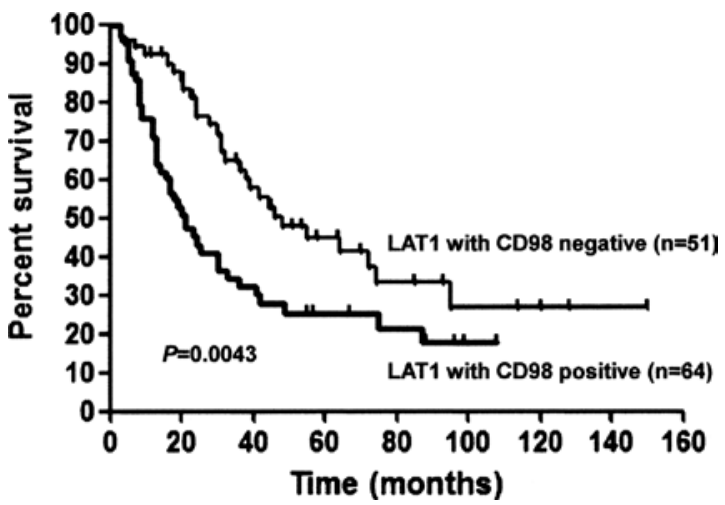

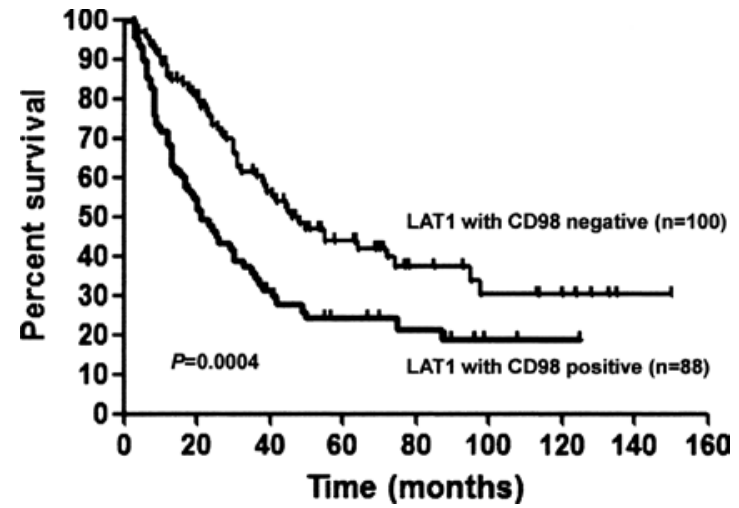

D

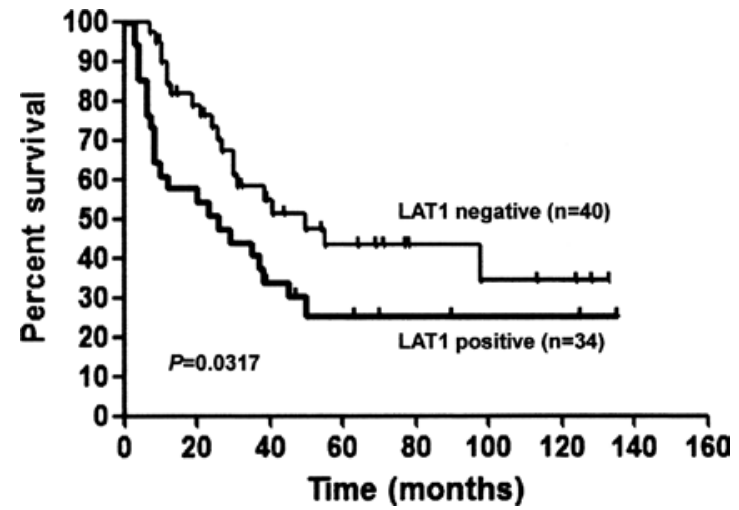

F

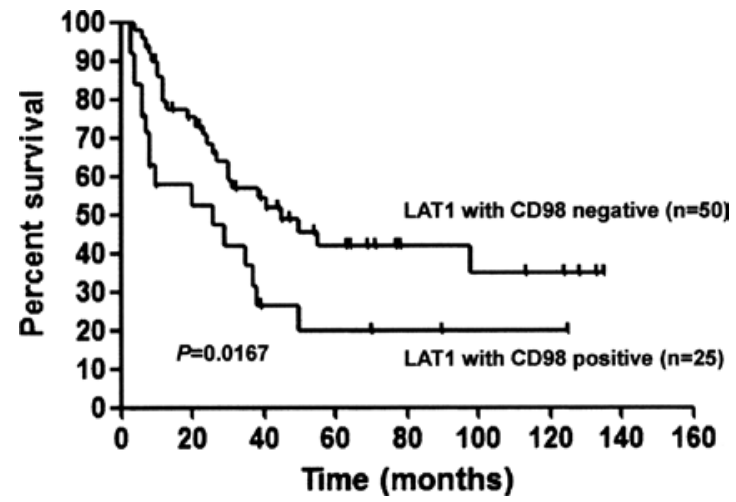

Figure 2. Postoperative survival of patients with completely resected pathologic stage III NSCLC. The comparison of postoperative survival rates was based on LAT1 expression and LAT1 with CD98 expression. The survival rate of LAT1-positive patients was significantly worse than that of LAT1-negative patients (A). The survival of LAT1 with CD98-positive patients was significantly worse than that of LAT1 with CD98-negative patients (B). LAT1 positivity was associated with an unfavorable prognosis in stage IIIA $(\mathrm{p}=0.0412)(\mathrm{C})$ and stage IIIB $(\mathrm{p}=0.0317)(\mathrm{D})$ patients. LAT1 with CD98-positivity was also associated with an unfavorable prognosis in stage IIIA $(\mathrm{p}=0.0043)(\mathrm{E})$ and stage IIIB $(\mathrm{p}=0.0167)(\mathrm{F})$ patients.

(30-32). L-[3 $\left.{ }^{18}-\mathrm{F}\right]$-methyltyrosine $\left({ }^{18} \mathrm{~F}-\mathrm{FMT}\right)$ was developed as a tracer for amino acid transporter using positron emission tomography (33). ${ }^{18} \mathrm{~F}-\mathrm{FMT}$ is transported via LAT1, and its uptake correlates with LAT1 expression in NSCLC $(27,30,34)$. A recent study demonstrated that the uptake of ${ }^{18} \mathrm{~F}$-FMT was significantly associated with CD98 expression, cell proliferation and angiogenesis (35). Future studies are warranted to clarify the association of ${ }^{18} \mathrm{~F}-\mathrm{FMT}$ uptake with prognosis and also with the cooperative expression of LAT1 and CD98 in NSCLC patients.

2-Aminobicyclo-(2,2,1)-heptane-2-carboxylic acid (BCH) is an amino acid-related compound that has been used as a selective inhibitor of the system L amino acid transporter, including LAT1 $(8,34)$. Kim et al described that inhibition of the amino acid transporter LAT1 by BCH led to apoptotic cell death in cancer cells by inducing intracellular deple- tion of essential neutral amino acids, such as L-leucine (36). Their experimental study suggested that LAT1 may be a new target for the suppression of the growth of cancer cells. Nawashiro et al also found that $\mathrm{BCH}$ inhibited the growth of C6 glioma cells in vitro and in vivo in a dose-dependent manner, and that treatment with $\mathrm{BCH}$ significantly improved the survival of rats inoculated with C6 glioma (17). Thus, LAT1 may be a molecular target for the therapy of human neoplasms.

Stage III NSCLC comprises a heterogeneous group of patients. The 1997 ISS is not sufficiently accurate to allow for the prediction of individual prognosis. Revision of the ISS has been proposed, having potential clinical implications. However, none of these revisions include novel biological markers. In the present study, LAT1 and CD98 expression appears to supplement prognostic information in the group 
Table V. Five-year survival according to the cooperative expression of LAT1 and CD98.

\begin{tabular}{|c|c|c|c|}
\hline \multirow[t]{2}{*}{ Variable } & \multicolumn{3}{|c|}{ Five-year survival rate $(\%)^{\mathrm{a}}$} \\
\hline & LAT1 with CD98-positivity & Other & p-value ${ }^{a}$ \\
\hline All patients & 24.1 & 43.6 & 0.0004 \\
\hline \multicolumn{4}{|l|}{ Age } \\
\hline$\leq 65$ years & 26.0 & 45.8 & 0.0269 \\
\hline$>65$ years & 22.8 & 46.2 & 0.0002 \\
\hline \multicolumn{4}{|l|}{ Gender } \\
\hline Male & 27.4 & 41.4 & 0.0299 \\
\hline Female & 15.4 & 50.1 & 0.0007 \\
\hline \multicolumn{4}{|l|}{ Pathologic stage } \\
\hline IIIA & 25.1 & 41.2 & 0.0043 \\
\hline IIIB & 19.6 & 42.0 & 0.0167 \\
\hline \multicolumn{4}{|l|}{ Histology } \\
\hline Adenocarcinoma & 19.4 & 39.7 & 0.0002 \\
\hline Squamous cell carcinoma & 26.4 & 61.5 & 0.0090 \\
\hline
\end{tabular}

a Kaplan-Meier analysis; blog-rank test. LAT1, L-type amino acid transporter 1.

Table VI. Multivariate analysis of the prognostic factors in stage III NSCLC.

\begin{tabular}{lccr}
\hline Prognostic factor & Hazard ratio & 95\% Confidence interval & p-value \\
\hline Age (s65 years/>65 years) & 0.809 & $0.381-1.716$ & 0.5798 \\
Gender (male/female) & 0.921 & $0.603-1.408$ & 0.7045 \\
Disease stage (IIIA/IIIB) & 0.914 & $0.607-1.378$ & 0.6692 \\
Histology (AC/non-AC) & 1.350 & $0.846-2.156$ & 0.2082 \\
LAT1 (positive/negative) & 1.529 & $1.053-2.219$ & 0.0255 \\
CD98 (positive/negative) & 2.118 & $1.140-3.934$ & 0.0175 \\
Ki-67 labeling index (low/high) & 1.531 & $0.839-2.794$ & 0.1652 \\
N factor (N0-1/N2-3) & 0.664 & $0.410-1.136$ & 0.1231 \\
\hline
\end{tabular}

Hazard ratios, $95 \%$ confidence intervals and two-sided p-values were obtained from the Cox proportional hazards models. LAT1, L-type amino acid transporter 1; AC, adenocarcinoma.

of patients with stage III NSCLC. Our study analyzed surgically resected tumors, while other studies have employed both biopsy and surgical samples $(37,38)$. Surgical samples are preferable for the immunohistochemical assessment of potential biological markers.

Patients with stage III NSCLC are a heterogeneous group that require multimodal therapy, including chemotherapy and/or radiotherapy. In this study, only patients with surgical resection were eligible. It is unknown whether LAT1 and CD98 expression is an independent prognostic factor in patients treated with chemotherapy and/or radiotherapy, and meta-analyses are needed to answer this question. Our results indicated that there was significant difference in LAT1 and CD98 expression between AC and SQC. Moreover, LAT1 expression alone was an independent prognostic factor in $\mathrm{AC}$, but not in SQC. Therefore, prognostic factors may differ according to the histological types of NSCLC.
This study had several limitations. The optimal treatment of stage III NSCLC has not been clearly defined, and many aspects of its therapy remain controversial. While there are many potential treatment options, none yield a high probability of cure. Additionally, the present study may not reflect the general population of stage III NSCLC, as the currently studied population had nonbulky stage III disease, and all patients were treated with surgical resection. However, the survival rate of our study was better than that reported in previous studies on stage III NSCLC $(36,37)$. Berghmans et al described that the undertaking of surgery is a significant independent prognostic factor for predicting a favorable prognosis in stage III NSCLC (36).

In conclusion, the expression of LAT1 and CD98 is a significant biological marker for predicting poor prognosis in patients with surgically resected stage III NSCLC. LAT1 expression was significantly correlated with CD98 expression, tumor cell proliferation and angiogenesis. The overexpression 
of LAT1 and CD98 plays an important role in the cellular proliferation and progression of NSCLC. The inhibition of LAT1 and CD98 function may in future serve as an effective therapeutic target for the treatment of stage III NSCLC.

\section{Acknowledgements}

The authors thank T. Hikino for technical assistance in the immunohistochemical staining of LAT1, Ki-67, CD98, VEGF, CD31 and CD34.

\section{References}

1. Shottenfeld D: Epidemiology of Lung Cancer. Lippincott-Raven, Philadelphia, PA, 1996.

2. Graziano SL: Non-small cell lung cancer: clinical value of new biological predictors. Lung Cancer 17 (Suppl 1): 37-58, 1997.

3. Mountain CF: Revision in the International System for Staging Lung Cancer. Chest 11: 1710-1717, 1997.

4. Andre F, Grunenwald D, Pignon JP, et al: Survival of patients with resected N2 non-small cell lung cancer: evidence for a subclassification and implications. J Clin Oncol 18: 2981-2989, 2000.

5. Leong SS, Rocha Lima CM, Sherman CA and Green MR: The 1997 International Staging System for non-small cell lung cancer: have all the issues been addressed? Chest 115: 242-248, 1999.

6. Jassem J, Skokowski J, Dziadziuszko R, et al: Results of surgical treatment of non-small cell lung cancer: validation of the new postoperative pathologic TNM classification. J Thorac Cardiovasc Surg 119: 1141-1146, 2000.

7. Clinical practice guidelines for the treatment of unresectable non-small cell lung cancer: adopted on May 16, 1997 by the American Society of Clinical Oncology. J Clin Oncol 15: 2996-3018, 1997

8. Christensen HN: Role of amino acid transport and countertransport in nutrition and metabolism. Physiol Rev 70: 43-77, 1990.

9. McGivan JD and Pastor-Anglada M: Regulatory and molecular aspects of mammalian amino acid transport. Biochem J 299: 321-334, 1994.

10. Oxender DL and Christensen HN: Evidence for two types of mediation of neutral amino acid transport in Ehrlich cells. Nature 197: 765-767, 1963.

11. Kanai Y, Segawa H, Miyamoto K, Uchino H, Takeda E and Endou H: Expression cloning and characterization of a transporter for large neutral amino acids activated by the heavy chain of 4F2 antigen (CD98). J Biol Chem 273: 23629-23632, 1998.

12. Yanagida O, Kanai Y, Chairoungdua A, et al: Human L-type amino acid transporter 1 (LAT1): characterization of function and expression in tumor cell lines. Biochim Biophys Acta 1514 291-302, 2001

13. Ohkame H, Masuda H, Ishii Y and Kanai Y: Expression of L-type amino acid transporter 1 (LAT1) and 4F2 heavy chain (4F2hc) in liver tumor lesions of rat models. J Surg Oncol 78: 265-272, 2001.

14. Kaira K, Oriuchi N, Imai H, et al: Prognostic significance of L-type amino acid transporter 1 expression in resectable stage I-III nonsmall cell lung cancer. Br J Cancer 98: 742-748, 2008.

15. Kaira K, Oriuchi N, Imai H, et al: Expression of L-type amino acid transporter 1 (LAT1) in neuroendocrine tumors of the lung. Pathol Res Pract 204: 553-561, 2008.

16. Nakanishi K, Ogata S, Matsuo H, et al: Expression of LAT1 predicts risk of progression of transitional cell carcinoma of the upper urinary tract. Virchows Arch 451: 681-690, 2007.

17. Nawashiro H, Otani N, Shinomiya N, et al: L-type amino acid transporter 1 as a potential molecular target in human astrocytic tumors. Int J Cancer 119: 484-492, 2006.

18. Chairoungdua A, Segawa H, Kim JY, et al: Identification of an amino acid transporter associated with the cystinuria-related type II membrane glycoprotein. J Biol Chem 274: 28845-28848, 1999.
19. Matsuo H, Tsukada S, Nakata T, et al: Expression of a system $\mathrm{L}$ neutral amino acid transporter at the blood-brain barrier. Neuroreport 11: 3507-3511, 2000.

20. Buck AC, Schirrmeister HH, Guhlmann CA, et al: Ki-67 immunostaining in pancreatic cancer and chronic active pancreatitis: does in vivo FDG uptake correlate with proliferative activity? J Nucl Med 42: 721-725, 2001.

21. Weidner N, Semple JP, Welch WR and Folkman J: Tumor angiogenesis and metastasis-correlation in invasive breast carcinoma. N Engl J Med 324: 1-8, 1991.

22. Mineo TC, Ambrogi V, Baldi A, et al: Prognostic impact of VEGF, CD31, CD34 and CD105 expression and tumor vessel invasion after radical surgery for IB-IIA non-small cell lung cancer. J Clin Pathol 57: 591-597, 2004.

23. Fucks BC, Finger RE, Onan MC and Bode BP: ASCT2 silencing regulates mammalian target-of-rapamycin growth and survival signaling in human hepatoma cells. Am J Physiol Cell Physiol 293: 55-63, 2007

24. Kaira K, Oriuchi N, Imai H, et al: L-type amino acid transporter 1 (LAT1) and CD98 expression in the primary site and the metastatic site of human neoplasms. Cancer Sci 99: 2380-2386, 2008.

25. Rintoul RC, Buttery RC, Mackinnon AC, et al: Cross-linking CD98 promotes integrin-like signaling and anchorage-independent growth. Mol Biol Cell 13: 2841-2852, 2002.

26. Esteban F, Ruiz-Cabello F, Concha A, Perez Ayala M, Delgado M and Garrido F: Relationship of 4F2 antigen with local growth and metastatic potential of squamous cell carcinoma of the larynx. Cancer 66: 1493-1498, 1990.

27. Kaira K, Oriuchi N, Otani Y, et al: Fluorine-18- $\alpha$-methyltyrosine positron emission tomography for diagnosis and staging of lung cancer: a clinicopathological study. Clin Cancer Res 13: 6369-6378, 2007.

28. Martin B, Paesmans M, Mascaux C, et al: Ki-67 expression and patient survival in lung cancer: systematic review of the literature with meta-analysis. Br J Cancer 91: 2018-2025, 2005.

29. Han H, Silverman JF, Santucci TS, et al: Vascular endothelial growth factor expression in stage I non-small cell lung cancer correlates with neoangiogenesis and a poor prognosis. Ann Surg Oncol 8: 72-79, 2001.

30. Oriuchi N, Higuchi T, Ishikita T, et al: Present role and future prospect of positron emission tomography in clinical oncology. Cancer Sci 97: 1291-1297, 2006.

31. Kaira K, Oriuchi N, Otani Y, et al: Diagnostic usefulness of fluorine-18- $\alpha$-methyltyrosine positron emission tomography in combination with ${ }^{18} \mathrm{~F}$-fluorodeoxyglucose in sarcoidosis patients. Chest 131: 1019-1027, 2007.

32. Inoue $\mathrm{T}$, Koyama K, Oriuchi $\mathrm{N}$, et al: Detection of malignant tumors: whole-body PET with fluorine-18- $\alpha$-methyl tyrosine versus FDG-preliminary study. Radiology 220: 54-62, 2001.

33. Tomiyoshi K, Amed K, Muhammad S, et al: Synthesis of new fluorine-18 labeled amino acid radiopharmaceutical: L-F-alphamethyl tyrosine using separation and purification system. Nucl Med Commun 18: 169-175, 1997.

34. Kim DK, Kanai Y, Choi HW, et al: Characterization of the system $\mathrm{L}$ amino acid transporter in T24 human bladder carcinoma cells. Biochim Biophys Acta 1565: 112-122, 2002.

35. Kaira K, Oriuchi N, Shimizu K, et al: Evaluation of thoracic tumors with ${ }^{18} \mathrm{~F}-\mathrm{FMT}$ and ${ }^{18} \mathrm{~F}-\mathrm{FDG}$ PET-CT: a clinicopathological study. Int J Cancer 124: 1152-1160, 2009.

36. Kim CS, Cho SH, Chun HS, et al: $\mathrm{BCH}$, an inhibitor of system $\mathrm{L}$ amino acid transporters, induces apoptosis in cancer cells. Biol Pharm Bull 31: 1096-1100, 2008.

37. Berghmans T, Meert AP, Martin B, Ninane V and Sculier JP Prognostic role of epidermal growth factor receptor in stage III non-small cell lung cancer. Eur Respir J 25: 329-335, 2005.

38. Berghmans T, Mascaux C, Martin B, Ninane V and Sculier JP: Prognostic role of thyroid transcription factor-1 in stage III non-small cell lung cancer. Lung Cancer 52: 219-224, 2006. 